José Manuel Gómez ; Celimar Rodríguez

\title{
Las competencias del profesor universitario en los entornos tecnológicos de información y comunicación
}

\section{The competences of the university teacher in the technological environments of information and communication}

\author{
José Manuel Gómez \\ josemanuelgog@gmail.com \\ Universidad Nacional Experimental Francisco de Miranda \\ Venezuela \\ Celimar Rodríguez \\ celirodel412@gmail.com \\ Universidad Nacional Experimental Rafael Maria Baralt \\ Venezuela
}

\section{RESUMEN}

El propósito de esta investigación es generar un perfil teórico sobre las competencias del docente universitario de la UNEFM, de los programas de educación en los entornos tecnológicos; su razón metodológica es cualitativa, caracterizada como una construcción social de carácter interpretativo. El tipo de investigación es descriptiva contextual siguiendo la Teoría fundamentada, según lo propuesto por Strauss y Corbin (2002). Los informantes fueron docentes del programa de educación de la UNEFM, desde la perspectiva haber desarrollado herramienta en los entornos tecnológicos, donde los datos que se recolectaron fueron a través de la entrevista. Para recolectar el captus; la entrevista abierta fueron guiados con la guía de preguntas como instrumento, y la experiencia del investigador en el área. El proceso analítico, para los microanálisis se realizaron a través de la diagramación codificada abiertamente, abierta axial y saturación para generar categorías que al relacionarlas, por medio del uso del software Atlas Ti, para construir el perfil teórico. La cual fue Tecno Pedagógica, que constituyo la teoría de las competencias educativas de los docentes universitarios en los entornos tecnológicos, se concluye que destaca dos elementos como son: 1) Tecnológico y 2) Pedagógico. La discusión de las categorías presentadas nos lleva a concluir que, si bien el desarrollo de las competencias universitarias es una temática que cobra especial relevancia en los últimos tiempos como consecuencia de su rápido 
CIENCIAMATRIA

Revista Interdisciplinaria de Humanidades, Educación, Ciencia y Tecnología

Año V. Vol. V. N8. Enero - Junio 2019

Hecho el depósito de ley: pp201602FA4721

ISSN-L: 2542-3029; ISSN: 2610-802X

Universidad Nacional Experimental Francisco de Miranda (UNEFM). Santa Ana de Coro. Venezuela

José Manuel Gómez ; Celimar Rodríguez

auge y probada utilidad de los entornos de ambiente virtual.

Palabras Claves: Perfil teórico, competencias, entornos tecnológicos.

\begin{abstract}
The purpose of this research is to generate a theoretical profile on the competencies of the university professor of the UNEFM, of the education programs in the technological environments; Its methodological reason is qualitative, characterized as a social construction of an interpretative nature. The type of research is descriptive contextual following the theory based, as proposed by Strauss and Corbin (2002). The informants were teachers of the UNEFM education program, from the perspective of having developed a tool in the technological environments, where the data that was collected was through the interview. To collect the captus; The open interview was guided with questions guide as an instrument, and the researcher's experience in the area. The analytical process for the microanalysis was done through the openly coded, axial open and saturation diagram to generate categories that by relating them through the use of Atlas $\mathrm{Ti}$ software to build the theoretical profile. It was concluded that Tecno Pedagógica, which constitutes the theory of the educational competences of university teachers in technological environments, highlights two elements: 1) Technological and 2) Pedagogical. The discussion of the categories presented leads us to conclude that, although the development of university competences is a topic that becomes particularly relevant in recent times as a consequence of its rapid rise and proven utility of virtual environment environments.
\end{abstract}

Keywords: Theoretical profile, competences, technological environments

\title{
IDEA IMPULSADORA DE LA INVESTIGACIÓN
}

En el nuevo escenario social del conocimiento, que adapta como elemento básico las tecnologías de la información uno de los requisitos básicos para participar en las nuevas formas de educar, para hacerle frente, es incorporando nuevos modelos de entornos de aprendizaje virtuales, que fomenten el trabajo activo y colaborativo. Desde esta perspectiva, para apoyar los procesos educativos, han surgido nuevas herramientas de convergencia alcanzando nuevo escenarios y nuevos lenguajes 
expresivos como propuestas innovadoras en las instituciones de educación universitaria, donde cada día se hace necesario que los docentes desarrollen habilidades y competencias en el uso educativo del computador y la red de redes: internet, diseño de contenidos multimedia, planificación didáctica adaptada a estos nuevos entornos tecnológicos y evaluación de los aprendizajes mediados con el computador.

En este sentido, una de las dificultades de la virtualidad en la educación, a nivel universitario, es de requerir docentes capacitados con nuevas destrezas laborales y una exigente preparación específica para enfrentar el tratamiento del proceso instruccional y dar respuestas a los nuevos desafíos del mundo globalizado en el campo social, tecnológico y educativo. Esta modalidad de estudios, le exige al docente nuevas competencias necesarias para utilizar las tecnologías de la información y la comunicación como herramientas para aprovechar el amplio abanico de posibilidades sin perder de vista los objetivos educativos y los entornos virtuales como espacio básicos de interacción.

Desde la perspectiva, el perfil del docente de la universidad, basado en la presencia de las Tecnologías de la información y la comunicación ha producido consigo unos cambios en el aprendizaje dinámico. El estudiante universitario, exigiéndole una manera de aprender este la autonomía, independencia, conectividad y la colaboración, propias de la condición de adulto, la cual se ve favorecida en la medida en que, como aprendiz, desarrolla sus habilidades y destrezas de pensamiento crítico para discernir, valorar el volumen de información al cual tienen acceso por medio de la conectividad a la red.

Esta conectividad permite el progreso de las tecnologías en la educación universitaria, permite poder implementar las TIC en el salón de clase, para promover el aprendizaje dinámico. Sin embargo, para que el aprendizaje dinámico mediante entornos tecnológicos sea exitoso, es fundamental y necesario las competencias que manejen los docentes universitarios; que cuenten con recursos tecnológicos, oportunidad de 
interacción entre los usuarios y miembros de la comunidad universitaria, redes o grupos colaborativos con objetivos similares.

La competencia profesional del docente, entendida más como una competencia intelectual, trasciende el sentido puramente técnico del recurso didáctico. Las competencias docentes se caracterizan por ser complejas: combinan habilidades, principios y conciencia del sentido y de las consecuencias de las prácticas pedagógicas; así como una reflexión y análisis sobre los contextos que las condicionan y que van más allá del aula. La competencia profesional "...se refiere no sólo al capital de conocimientos disponibles, sino a los recursos intelectuales de que dispone con objeto de hacer posible la ampliación y desarrollo de ese conocimiento profesional, su flexibilidad y profundidad" (Contreras, 1999:58).

Al mismo tiempo, las herramientas tecnológicas facilitan un progresivo componente informal que se integra con los sistemas formales. Así van brotando nuevos espacios que permiten la posibilidad de comunicación con una gran variedad, como lo son: entornos institucionales, campus virtuales, entornos virtuales, espacios informales, redes sociales, entornos personales, entre otros. Entornos que tanto profesores como estudiantes miembro de una comunidad universitaria deben dominar y apropiarse para que se genere el aprendizaje dinámico con la construcción personal del conocimiento, la realidad del conocimiento partiendo de las experiencias personales.

La UNESCO (2009) plantea que la educación superior debe ampliar la formación de docentes, como en el empleo, con planes y programas de estudios que den a los docentes la capacidad de dotar a sus alumnos de los conocimientos y las competencias que necesitan en el siglo XXI. Este objetivo plantea nuevos enfoques, como el uso del aprendizaje abierto, a distancia y de las tecnologías de la información y la comunicación (TIC). Esta tecnología, constituyen hoy en día un aliado para la educación, a través de herramientas innovadoras que permitan al profesor la adquisición de destrezas, competencias y habilidades que coadyuven a desenvolverse en el contexto en el cual está inmerso. Las herramientas tecnológicas están vinculadas con la implementación 
de nuevas estrategias interactivas, las cuales puedan desempeñar un papel relevante en el campo educacional como estadio de desarrollo social del conocimiento.

La Competencia digital implica el uso crítico y seguro de las Tecnologías de la Sociedad de la Información para el trabajo, el tiempo libre y la comunicación. Apoyándose en habilidades TIC básicas: uso de ordenadores para recuperar, evaluar, almacenar, producir, presentar e intercambiar información, y para comunicar y participar en redes de colaboración a través de Internet (Comisión europea, 2012).

La formación que ofrecen los establecimientos de enseñanza superior debería atender las necesidades sociales. Esto comprende la divulgación de la investigación para elaborar y aplicar nuevas tecnologías y garantizar la educación universitaria. Con esta sociedad de la información y comunicación, los docentes deben desarrollar habilidades integrar para las tecnologías de información y comunicación, como medio instrumental y didáctico, desarrollar destrezas cognitivas y el esfuerzo crítico para aprender, desaprender y reaprender desde las tendencias actuales la relación emergente en esta sociedad del conocimiento.

Nos encontramos en una sociedad donde el aprender a aprender es de máxima importancia, en Venezuela, el Plan Nacional "Simón Bolívar" deja por sentado que el Gobierno revolucionario asume la responsabilidad de la educación en todos los niveles, con lo que realmente se garantiza el acceso libre y gratuito a las instituciones educativas públicas del país. Permite profundizar la universalización de la educación bolivariana, donde fortalece e incentiva la investigación en proceso educativo, incorpora las tecnologías de información y comunicación al proceso educativo (Proyecto Nacional Simón Bolívar, 2007: 12).

Estamos por tanto hablando de un elemento básico de tecnología de información para el desarrollo y potencian del conocimiento con pertinencia, inclusión y participación en el sistema educativo; en particular el sistema educativo universitario, caracterizado por la capacidad de sus miembros para obtener, compartir y procesar cualquier información por medio telemático desde cualquier lugar y en la forma que se prefiera. La Educación 
Universitaria en Venezuela, debe responder a los requerimientos actuales de la sociedad del conocimiento, y sustentarse en los avances científicos, tecnológicos y comunicacionales.

Su desarrollo ha de favorecer la inclusión en el Sistema de Educación Universitaria; además, debe garantizar su calidad en términos de su eficacia, eficiencia y pertinencia; en su carácter más definido con la aprobación de la nueva Ley Orgánica de Educación, la cual establece en su artículo 33 que "la educación universitaria tiene como principios rectores fundamentales los establecidos en la Constitución de la República, el carácter público, calidad y la innovación, el ejercicio del pensamiento crítico y reflexivo, la inclusión, la pertinencia, la formación integral” (Ley Orgánica de Educación, 2009).

Existe una propuesta de Proyecto Nacional de Educación Universitaria a Distancia, realizado por el Consejo Nacional de Universidades y la Oficina de Planificación del Sector Universitario (2009) donde ofrece lineamientos y pautas que permiten garantizar un desarrollo ordenado de la modalidad a distancia, en el ámbito de la educación superior venezolana, a fin de alcanzar los niveles académicos de calidad requeridos en la sociedad del aprendizaje en la que la adquisición del conocimiento no está relegada a instituciones presenciales.

Para favorecer la inclusión en igualdad de oportunidades para la formación y actualización a nivel superior ampliando la cobertura espacial y temporal, y atendiendo las necesidades educativas especiales de los estudiantes.

Teniendo en cuenta la transformación, cambio y reto que esta esta sociedad de la información origina, nos lleva a buscar respuesta con el desarrollo de Internet conocido como Web 2.0, que se viene progresivamente desarrollando en la comunidad universitaria, está potenciando una nueva manera de interactuar y compartir en la Red: presentando nuevas herramientas que promueve la dimensión social de la Red; estimulando la arquitectura de la participación; y orientada a la interacción.

Como lo afirma Peña, Pérez y Rondón (2010) sobre "el desarrollo exponencial y el amplio espacio que vienen tomando las redes sociales, apoyadas en las Tecnologías 


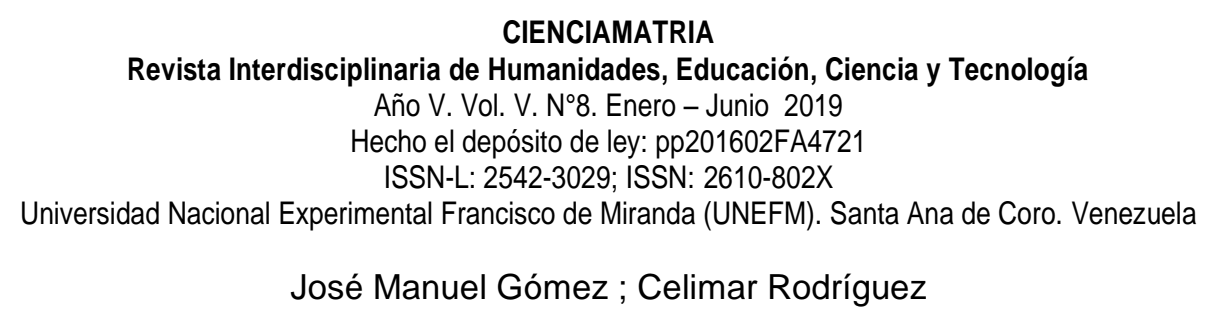

de Información y Comunicación (TIC), especialmente por el atractivo que tienen para los usuarios de Internet, quienes pueden expresar sus ideas libremente, una serie de actividades en línea que procuran la socialización, la colaboración y el encuentro" p.176. Esto se debe precisamente a la Web 2.0, y su característica en lo social, porque se ha favorecido la conformación de comunidades virtuales y redes de colaboración entre pares. (Cobo y Romaní, 2007: 84).

Las tendencias en educación universitaria adoptan el trabajo en grupo, en la cual "los alumnos son los protagonistas del trabajo en el aula. La interacción que se produce en el aula no sólo es la de profesor-grupo. Así mismo, (Gutiérrez, Rodríguez e Isea, 2017), señalan que

Para el nuevo siglo, la propuesta innovadora que actualmente está en boga es el enfoque por competencias o currículo basado en competencias, cuyo empleo se empieza a generalizar en el ámbito educativo, donde su acción comienza principalmente en los procesos de reforma de los planes de estudio. (p. 233).

Es fundamental también tener en cuenta la interacción entre el alumno y el profesor y la de los alumnos entre sí. En múltiples ocasiones los estudiantes aprenden más de sus compañeros (del compañero experto) que del propio profesor, basado en un aprendizaje dinámico" (Ayerdi, Pérez y Mendiguren, 2010)

Estos avances en el acceso a recursos y materiales para el aprendizaje, se presentan con nuevas maneras y situaciones comunicativas en lo personal y virtual. Estos espacios de relación social, se buscan un nuevo aprendizaje interactivo, conectivo; entre docente y alumno. Estas nuevas aplicaciones con la tecnología permiten a los usuarios en gestores de unos contenidos donde juegan un papel crucial en el ámbito educativo universitario. Son una de las estructuras sociales más potentes e innovadoras para el trabajo en red (Santamaría, 2008) y han propiciado la creación de comunidades de aprendizaje y redes de conocimiento.

La impactante entrada de nuevas plataformas de comunicación social, conocidas como entornos tecnológicos, ha hecho que la Universidad Nacional Experimental "Francisco 


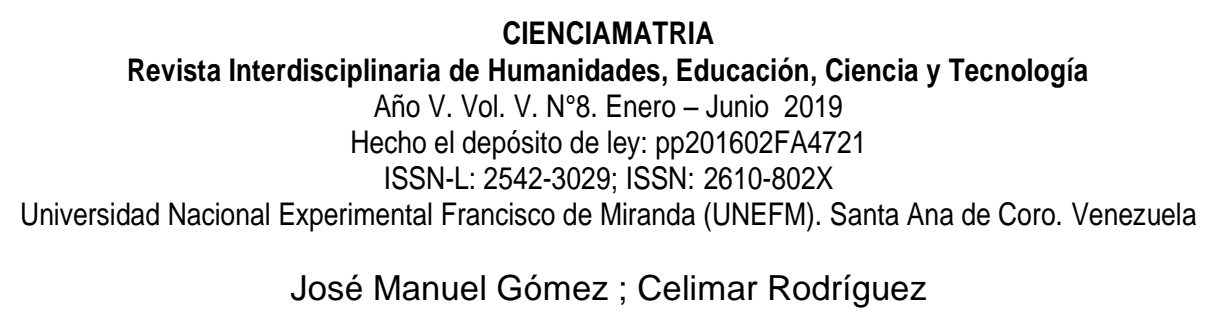

de Miranda" de la Ciudad de Coro, estado Falcón; se observa una tendencia entre una relación emergente que está sucediendo con el uso de la modalidad de Aprendizaje Dialógico Interactivo (ADI); donde Fundamentado en un modelo curricular integral comprehensivo, ADI se basan en un enfoque de enseñanza-aprendizaje centrado en el alumno y en los grupos colaborativos.

Toda esta situación, nos hace pensar cómo se puede interpretar la concepción filosófica que está sucediendo en las competencias del docente universitario, con su uso y aplicación de entornos tecnológico, en la Universidad Nacional Experimental "Francisco de Miranda" (UNEFM).

Partiendo de una interpretación, donde se categoricen las competencias del docente universitario en relación a los entornos tecnológicos en la UNEFM; en el que se realice una recogida de datos del uso de la comunidad académica universitaria de la UNEFM, se codifican esos datos y se reflexionan analíticamente. Para elaborar los lineamientos teóricos acerca de las competencias del docente universitario, donde es fundamental que se descubran, construyan y relacionen las categorías encontradas que constituyen un elemento conceptual para derivar los fundamentos teóricos relacionados con los entornos tecnológicos.

\section{Propósito General}

Generar un perfil por competencias del docente universitario en los entornos tecnológicos

\section{Propósitos Específicos}

- Categorizar las proposiciones teóricas que emergen como categorías de los significados que le atribuyen los actores participantes a las competencias del docente universitario para desarrollar tecnología en la instrucción

- Relacionar a través del microanálisis de categorías y propiedades desde la definición de los actores participantes las competencias docentes en los entornos tecnológicos para el aprendizaje 
- Integrar y refinar la teoría en desarrollo sobre las competencias del docente en los entornos tecnológicos para el proceso de enseñanza y aprendizaje en la UNEFM

\section{PARADIGMA DE INVESTIGACIÓN}

En esta investigación se adopta los parámetro de la investigación cualitativa en donde se describe un paradigma interpretativo, se estudiaron las competencias del docente universitario y se analizó la relación entre los actores sociales involucrado con su realidad pedagógica y su relación con los entornos tecnológicos en la Universidad Nacional Experimental "Francisco de Miranda".

Este paradigma en la relación sujeto objeto es de interacción hasta el punto que se influyen, siendo el investigador, sujeto de investigación e informante clave "Sujeto interactivo" comunicativo que "comparten significados" (Strauss y Corbin, 2002), constituye una reflexión desde la praxis. La realidad está constituida no sólo por hechos observables y externos, sino también por significados simbólicos e interpretaciones elaboradas por el sujeto a través de la interacción con los entornos tecnológicos.

La investigación es de tipo descriptiva contextual, porque parte del hecho que existe una realidad que resulta no conocida y al mismo tiempo interesante para ser interpretada y proveer un buen registro del individuo como un sujeto interactivo, comunicativo, que comparte significados. Donde la concepción del alumno del conocimiento está influida por la realidad que le rodea. El alumno debe conocer dentro de un medio real y un contexto social que hay que comprender e interpretar, basado en la conectividad de las redes sociales.

\section{MÉTODO DE INVESTIGACIÓN}

En relación al método de investigación, se empleó la teoría fundamentada (Strauss y Corbin, 2002), donde nos permitió la Integrar y refinar la aproximación teórica de la práctica que conforma las competencias del docente universitario en los entornos 
tecnológicos con los constructos teóricos que los fundamenten a partir de los significados de los actores implicados en la UNEFM. Para ello, se partió de la recolección de datos, el análisis de los datos y la generación de la teoría.

El objetivo de la teoría fundamentada, es descubrir una teoría que logre explicar a partir de la inducción un suceso a partir de incidentes derivados del Campo de estudio, en nuestro caso fue los docentes de la UNEFM.

Strauss y Corbin, 2002, plantea los procedimientos en la teoría fundamentada en los siguientes pasos: 1. recogida de datos, 2 . codificación y 3. reflexión analítica en notas. Es importante que se descubran, construyan y relacionen las categorías encontradas; estas constituyen el elemento conceptual de la teoría y muestran las relaciones entre ellas y los datos.

La investigación se estructuro en las etapas siguientes:

1. Definición de las preguntas de investigación: Esta etapa correspondió a la definición de las preguntas básicas de la investigación, las cuales fueron precisas que permitieron focalizar la investigación y la flexibilidad de explorar el fenómeno con profundidad, todo ello siguiendo las indicaciones que exponen Strauss y Corbin (2002).

2. Decisiones de muestreo. Una vez que fueron generadas las preguntas básicas y la investigación fue focalizada, se procedió a seleccionar el primer caso de acuerdo al principio de muestreo teórico, el cual plantea la selección de los contextos y casos porque tienen uno o varios atributos que ayudan a ir desarrollando la teoría. En el caso que nos ocupó se tomó como contexto inicial para la investigación el programa de Educación de la Universidad Nacional Experimental Francisco de Miranda, ubicado en la ciudad de Coro del estado Falcón, satisfaciendo el criterio de heterogeneidad, con la diversidad de actores sociales que conforman la institución y el de accesibilidad (facilidad de aprovechar los recursos disponibles de la institución).

El proceso de recolección de datos se desarrolló siguiendo el principio del muestreo teórico el cual también indicó como una vez recolectados, codificados y analizados los datos se decidió cual dato debió seguirse recolectando y donde encontrarlo a medida 
que se fue desarrollando la investigación y fue emergiendo la teoría y cuando en la recolección de datos fueron emergiendo las principales categorías, se fueron cubriendo en profundidad solamente los datos necesarios que subsecuentemente se requirieron de cada categoría para el desarrollo de sus propiedades y proposiciones.

El criterio para considerar cuando hubo de detener el muestreo teórico fue la saturación de la categoría o también denominada saturación teórica, la cual se refiere a la situación en la cual no se encuentran datos adicionales con los cuales el investigador pueda desarrollar propiedades de la categoría y a medida que observa situaciones similares una y otra vez, llega a estar empíricamente confiado que la categoría está saturada y no queda más que continuar a un nuevo grupo para recolectar datos de otras categorías e intentar saturarlas también, tal y como lo menciona Panditt (1995).

3. Selección de estrategias de obtención, análisis y presentación de los datos: Para este momento de la investigación se asumió la codificación axial donde se reagruparon los datos que se fracturaron durante la codificación abierta, aquí las categorías se relacionaron con sus subcategorías para formar explicaciones más precisas y completas sobre los fenómenos, tomando en consideración que una categoría representa un fenómeno, un fenómeno tiene la capacidad de explicar lo que sucede, una subcategoría también es una categoría pero en lugar de representar al fenómeno responde preguntas sobre los fenómenos tales como cuándo, dónde, por qué, quién, cómo y con qué consecuencias, dando así a los conceptos un mayor poder explicativo (Strauss y Corbin, ob. cit.).

Igualmente se aplicó la codificación selectiva que fue el proceso de integrar y refinar la teoría lo cual involucró la integración de las categorías que fueron desarrolladas organizándolas alrededor de un concepto explicativo central. Comenzó con la generación de una línea narrativa que vino a ser la conceptualización de la descripción acerca del fenómeno, la cual al ser analizada se convirtió en una categoría central. Una vez que se estableció el compromiso con esta categoría central, el resto de las 
categorías subsidiarias se relacionaron con ella por medio de las oraciones que explican las relaciones.

4 Examen y Comparación con la literatura existente: Seguidamente a la generación del marco teórico relacionado con el primer caso, se procedió a examinarlo y desarrollarlo a través de la selección de casos adicionales de acuerdo al principio de muestreo teórico, con el propósito de extender y/o afinar la teoría emergente hasta que el valor marginal de los nuevos datos fue mínimo y se cumplió con el principio de saturación teórica. Aquí se decidió el cierre del proceso y se continuó con la actividad de comparar la teoría emergente con la literatura existente, examinando lo que es similar o diferente y por qué. Este paso es muy importante porque realza la validez interna, la generalización y el nivel teórico de la construcción de teoría (Panditt, ob. cit.).

\section{Informantes clave}

La selección de los informantes claves, estuvo orientada por la reflexión de los siguientes criterios:

Haber aprobado el curso de FEDITIC

Tener carga académico en la modalidad de ADI

Haber desarrollado una herramienta en los entornos tecnológicos.

Cuadro 1

Caracterización de los Informantes

\begin{tabular}{ccc}
$\begin{array}{c}\text { Categoría de } \\
\text { escalafón } \\
\text { universitario }\end{array}$ & $\begin{array}{c}\text { Departamento } \\
\text { adscrito }\end{array}$ & Cantidad \\
\hline Asistente & Idiomas & $\mathbf{1}$ \\
Agregado & Práctica Profesional & $\mathbf{1}$ \\
Agregado & Física y Matemática & $\mathbf{1}$
\end{tabular}




\section{Total}

Fuente: (Gómez, 2017)

\section{Técnica empleada para la Recolección de la información}

Para la recolección de datos se utilizó la técnica de la entrevista en profundidad no estructurada, será utilizada, siguiendo a Martínez (1991), con el propósito de develar e interpretar, a través de diálogos orientados al foco de interés del estudio, persiguiendo en todo momento a conocer y entender las experiencias y por interrogantes abiertas analíticos procesos vividos por los actores implicados en los entornos tecnológicos.

Los datos recolectados tanto en la revisión documental y entrevistas en profundidad fueron convertidos en transcripciones digitalizadas y posteriormente transformados en documentos de texto que luego fueron incluidos como documentos primarios en el paquete de software para análisis de datos cualitativos denominado ATLAS/ti, el cual es un programa para codificar datos y construir teoría fundamentada. Este programa facilitó las tareas de codificación y categorización mediante la cual se desarrolló la etapa de reducción de los datos.

El programa ATLAS/ti permitió crear una Unidad Hermenéutica para el procesamiento de los datos en donde desde el inicio fueron emergiendo etiquetas conceptuales producto de la reducción de datos generada del análisis textual de los documentos primarios, este análisis o microanálisis como lo denominan Strauss y Corbin (2002) incluye la codificación abierta y la codificación axial, exige examinar e interpretar los datos de manera cuidadosa y minuciosa, realizando para ello una fragmentación de los datos en citas y trabajando con ellas.

El análisis inició con la comparación de los datos, a partir de la interpretación del investigador acerca del mundo de los participantes. En las respuestas se identificó la 
idea principal y se generó un código. Además, se realizó un análisis de afinidad semántica, que consistió en verificar en un diccionario y glosario los significados usuales de las palabras que aparecían en esas ideas principales, se contrastaron sus significados y se generaron los códigos.

En el caso de los atributos no deseables, se ubicó el código en un primer momento, para después encontrar el sinónimo con la ayuda de un diccionario, de tal manera que pudiera hablarse de presencia de atributos y no de su ausencia.

Durante esta etapa de la codificación abierta consistió en dividir y codificar los datos en conceptos y categorías. Durante esta etapa de análisis, el investigador codifica los distintos incidentes en categorías. Esta fragmentación que generó un total de noventa y cinco (95) citas se muestra en un informe que arrojó el programa.

Seguidamente las citas fueron comparadas y agrupadas por similitud de propiedades y dimensiones dándoles la misma etiqueta conceptual o código como se denomina en el programa ATLAS/ti, resultando la lista de códigos que se refleja en el Cuadro 2.

\section{Cuadro 2}

Codificación de los datos cualitativos

\section{Códigos}

№ de citas relacionadas en el código

Característica docente

Entornos tecnológicos

Entornos Virtuales

Estrategias virtuales

Competencias virtuales

Practicas Pedagógicas
18

17

11

23

7

19

95

Fuente: (Gómez, 2017) 


\section{CIENCIAMATRIA \\ Revista Interdisciplinaria de Humanidades, Educación, Ciencia y Tecnología \\ Año V. Vol. V. N8. Enero - Junio 2019 \\ Hecho el depósito de ley: pp201602FA4721 \\ ISSN-L: 2542-3029; ISSN: 2610-802X \\ Universidad Nacional Experimental Francisco de Miranda (UNEFM). Santa Ana de Coro. Venezuela \\ José Manuel Gómez ; Celimar Rodríguez}

A cada uno de estos códigos corresponde una definición que resultó de examinar el contenido de los tópicos que aparecen en los fragmentos o citas.

En lo que respecta al procedimiento de análisis, éste tuvo por finalidad obtener evidencias en torno a las categorías y significados asociados de manera teórica y evidencias construidas a partir de las categorías y temas obtenidos de los distintos actores así como también de los datos derivados del proceso analítico de los documentos. (Glaser y Strauss, 2002).

\section{HALLAZGOS}

La metodología que se empleó para esta investigación permitió que cada propósito específico que se estableció estuviera dado por las codificaciones de la teoría fundamentada (Codificaciones abierta, axial y selectiva), lo que permitió construir la teoría que generó la competencia del docente universitario en entornos tecnológicos. En cuanto a los elementos que constituirían la teoría de las competencias educativas de los docentes universitarios en los entornos tecnológicos, se concluye que esta tesis destaca la palabra Tecno pedagógica.

En cuanto al término "tecno pedagógica" en la consulta bibliográfica y por la web, sobre la existencia de este término, se evidencia como elemento dentro de algunos herramientas pero no como teoría emergente solo como una metodológica para la producción ágil de objeto de aprendizaje.

Con la intención de describir las conclusiones se abordaron atendiendo a los propósitos específicos. Primeramente el relacionado a categorizar las proposiciones teóricas que emergen como categorías de los significados que le atribuyen los actores participantes a las competencias del docente universitario para desarrollar tecnología en la instrucción que permitieron su comprensión y explicación sobre la realidad del profesorado universitario, donde ha experimenta numerosos métodos y técnicas de enseñanza-aprendizaje, elaborando experiencias y prácticas formativas, creadoras de conocimientos y emociones nuevas en estos ambientes virtuales. 
Podemos mencionar que uno de los elementos primordial generado como categoría que es el referido a pedagógica donde se basa en referencia a la capacidad para expresarse, establecer contacto y relacionarse en espacios virtuales a través de diversos medios, de manera sincrónica y asincrónica, es la conexión entre estudiantes, docentes, investigadores y miembros de la comunidad universitaria, donde permiten conectarse con datos, recursos, redes y experiencias de aprendizaje.

La pedagogía es una realidad eminentemente necesaria en los docentes universitarios en los entornos tecnológicos, se considera la articulación, la organización de la expresión y del discurso como la estrategia del contexto educativo. En general, la categoría consiste en un conjunto de conocimientos, herramientas y capacidades donde subyacentes al uso de la información que le permite a un docente transferir el proceso de enseñanza en los entornos virtuales. El enfoque pedagógico de ADI que se utiliza esta consecuente y muy alineado con todos los componentes del proceso instruccional propuesto en sus premisas, y entre ellos las decisiones acerca de la enseñanza en contextos virtuales.

Otro elemento que emergió de la categorías de los significados que le atribuyen los actores participantes, es la didáctica virtual, es la manera y forma que los docentes diseñan, proponen y conducen situaciones en ambientes virtuales que plantean a los estudiantes para la toma de decisiones razonadas y críticas, sobre la solución de problemas académicos e inclusive personales.

Esto de contener en ámbitos para su correcta elaboración, que corresponda al área de conocimiento de la materia o asignatura, la organización de contenidos educativos e informativos, así como en las tecnologías para el aprendizaje y en los elementos de comunicación de apoyo al aprendizaje del estudiante.

Esta categoría que brotó permite facilitar la intencionalidad y de manera progresiva que el alumno dirigidas al logro de las habilidades de autodirección, tales como recuerdo de información, establecimiento de objetivos, pensamiento crítico, autogestión y 
autoevaluación, promoviéndose así actitud esencial de los alumnos que participan en los entornos tecnológicos.

Otra categoría presente es lo relacionado a herramientas tecnológicas donde se establece los contextos virtuales que se basa los entornos tecnológicos en el cual deben estar orientada al aprendizaje, deben ser significativas y desarrollar las habilidades deseadas dentro del diseño de contenidos.

La plataforma utilizada en ADI UNEFM plantea herramientas que permite la evaluación en línea, donde se desarrollan la habilidad de alto nivel como pensamiento crítico y solución de problemas; incluye identificar, describir y relatar. Cuando se evalúan por los entornos tecnológicos, se realiza mediante videos que muestran presentaciones, debates mediante el foro y construcción de conceptos por medio de wiki, listas de significado por los glosarios; así como posters y otras presentaciones mediante el envío de tareas y adjuntos de ensayos.

Por otro lado, las competencias comprende la divulgación de la investigación para elaborar y aplicar nuevas tecnologías y garantizar la educación universitaria. Con esta sociedad de la información y comunicación, los docentes universitarios deben desarrollar habilidades que integren las tecnologías de información y comunicación, como medio instrumental y didáctico, desarrollar destrezas cognitivas y el esfuerzo crítico para aprender, desaprender y reaprender desde las tendencias actuales la relación emergente en esta sociedad del conocimiento.

En referencia al segundo propósito donde se relaciona a través del microanálisis de categorías y propiedades desde la definición de los actores participantes las competencias docentes en los entornos tecnológicos para el aprendizaje que están presente en la teoría Tecno Pedagógica se considera el diseño de contenido, referido a la planificación sistemática y estructurada en la producción de material didáctico adecuada a las necesidades de aprendizaje de los estudiantes y está en coherencia con un modelo educativo virtual. 
Los diseños de contenidos, debe basarse en los criterios de selección, los cuales se presenta a continuación:

- Deben ser adecuados: conviene determinar su propiedad del espacio educativo y el área donde se utilizará el contenido diseñado.

- Cumplí con el diálogo interactivo. Los elementos del currículo deben ser adaptados a las tecnologías de conectividad dialógica con intención de que la información a presentar sea de forma comprensible y usable por todo el alumnado a que va dirigido; esto es fundamento de la modalidad ADI y tecnológico: que no sea necesario disponer de unas condiciones tecnológicas extraordinarias de software, equipos, dispositivos y periféricos. El contenido diseñado debe permitir al alumno tomar decisiones y apreciar las consecuencias de las mismas.

- Tener una necesidad. El diseño de un contenido debe surgir de una necesidad. Puede ser la falta de motivación de algunos alumnos/as hacia determinados contenidos o la necesidad de hacer algo distinto o más eficaz con ellos son justificaciones legítimas para buscar un contenido desarrollados virtualmente para asegurar una mayor transferencia.

En cuanto a otro elemento generado en la teoría está referido a la evaluación en línea, esta concerniente a los procedimientos, herramientas y formas de evaluar los conocimientos mediante procesos descriptivos o conceptuales (exámenes, pruebas de destreza, problemas teóricos) en medios virtuales. Esto consiste en diseñar los objetivos, contenidos, competencias y criterios de evaluación que se pretenden desarrollar en el contenido instruccional. La fuente inicial debe ser el diseño instruccional del programa de la UNEFM. De igual modo, la concreción de un sistema de evaluación en línea debe ser coherente con los objetivos y contenidos desarrollados como competencia de diseño de contenidos en relación con la asignatura debe estar adecuada para constatar el logro de los propósitos y el nivel de asimilación de los contenidos de los estudiantes. 
El proceso de evaluación a distancia es efectivo y fiable cuando presenta aspectos cuantitativos y cualitativos, una evaluación sumativa y formativa, a través de distintos recursos que no sólo busquen informar al alumno sobre el progreso, sino que también beneficien el aprendizaje.

La discusión de las categorías presentadas como generación teórica nos lleva a concluir que, si bien el desarrollo de las competencias universitarias de los docentes en entornos tecnológicos es una temática que cobra especial relevancia en los últimos tiempos como consecuencia de su rápido auge y probada utilidad de los entornos de ambiente virtual.

El último propósito desarrollado donde se integra y refina la teoría en desarrollo sobre las competencias del docente en los entornos tecnológicos para el proceso de enseñanza y aprendizaje en la UNEFM, donde nace el modelo tecno pedagógico conlleva la conectividad y a la interactividad, lo cual requiere manejo en los entornos tecnológicos, donde posibilita la confluencia de la didáctica virtual. Así, la interactividad tiene sentido cuando los sujetos del acto educativo se benefician de ella a través de los flujos de información y conocimiento que se transmiten vía plataformas tecnológicas.

El uso de ATLAS TI en general la teoría fundamentada sobre Tecno Pedagógica aplicando los planteamientos metodológicos de Glaser y Strauss. Este software permitió expresar el sentido circular del análisis cualitativo, por cuanto otorga la posibilidad de incorporar secuencialmente los datos, sin la necesidad de recoger todo el material en un mismo tiempo. Por esta razón, permitió llevar a cabo el muestreo teórico necesario para realizar el análisis constructor de teoría.

Asimismo, ATLAS TI permitió identificar aquellos códigos que requieren ser saturados, esto es posible a través de la función code-primary-documents-table (códigos documentos primarios-tablas), que muestra la cantidad de citas que cada código tiene. Por tanto, esta función del software facilita la aplicación de la saturación de contenido de cada código y categoría, tal como se propone en la Teoría Fundamentada. Al igual que la generalidad de los programas informáticos, ATLAS TI es un programa de 
recuperación de texto, sin embargo está dirigido a un trabajo conceptual, en donde cada paso de la codificación teórica (codificación abierta, axial y saturación) tiene un espacio en el programa.

En tanto, la codificación axial tiene su espacio con la función de creación redes de relaciones conceptuales. Son esquemas explicativos, pero no jerárquicos, de las categorías y sus subcategorías en función de las relaciones entre familias de códigos que permitió elaborar una categoría central que integra los códigos y categorías construidos en las fases de codificación abierta y axial, la cual fue Tecno Pedagógica.

El resultado de lo presentado por ATLAS/ti nos visualizar validar el modelo Tecno Pedagógica de acuerdo con la evidencia obtenida a través de las diferentes perspectivas de los actores, toda vez que los diferentes docentes sustentan, justifican y explican el requerimiento de conectarse por medio de los entornos tecnológicos, donde existan las herramientas de comunicación e interacción con los miembros de la comunidad universitaria en conexión en tiempo real.

Presentar didáctica de gestión educativa centrados en el aprendizaje de los alumnos, lo cual implica fomentar el estudio independiente y el estudio en colaboración; la interacción académica entre profesor-alumno, alumno-profesor; el desarrollo de habilidades cognoscitivas en los estudiantes y el fomento de su capacidad de análisis, de síntesis y de formulación de juicios valorativos. En consecuencia, se requiere esta competencia del docente universitario donde debe incidir en aprendizajes, diseñar estrategias de aprendizaje individual y en equipo que fomente el análisis crítico y la reflexión como bases fundamentales del trabajo en colaboración. En este sentido, los docentes universitarios deben diseñar estrategias para recrear los conocimientos por medios de los materiales de instrucción y el fomento del aprendizaje colaborativo mediante la asignación de proyectos que generen discusiones en equipos. 


\section{REFERENCIAS CONSULTADAS}

1. Ayerdi, Pérez y Mendiguren, (2010) Experimentando con las redes sociales en la enseñanza universitaria en ciencias.

2. CONTRERAS, J (1999), La autonomía del profesorado, Madrid, Morata.

3. COLEMAN, J. (1988). "Social Capital in the Creation of Human Capital". The AmericanJournal of Sociology, Vol. 94, Supplement: Organizations and Institutions: Sociological and Economic Approaches to the Analysis of Social Structure.

4. COBO, C.; PARDO, H. (2007). Planeta Web 2.0. Inteligencia colectiva o medios fastfood. Grup de Recerca d'InteraccionsDigitals, Universitat de Vic. Flasco México. Barcelona / México DF.

5. COELLO, Y (2010) Procesos de las innovaciones curriculares, desde los actores

6. Comisión Europea (2012). Marco Común de Competencia Digital Docente.

7. Delors, J. (Coord.) (1996). La educación encierra un tesoro. Informe a la UNESCO de la Comisión Internacional sobre la educación para el siglo XXI. Madrid, España: Santillana. Ediciones UNESCO.

8. GARCÍA, I (2009). La teoría de la conectividad como solución emergente a las estrategias de aprendizaje innovadoras. Universidad Autónoma de Madrid UAM. Madrid.

9. GUTIÉRREZ, N., RODRíGUEZ, N., \& ISEA, J. (2017). LA EMOCIONALIDAD FRENTE A LA INNOVACIÓN CURRICULAR. Revista Arbitrada Interdisciplinaria Koinonía, 2(3), 226-246. Recuperado de http://fundacionkoinonia.com.ve/ojs/index.php/revistakoinonia/article/view/61/48

10. GLASER, B., y STRAUSS, A. (1967). Discovery of grounded theory. Chicago: Aldine

11. Flick, U. (2007). Introducción a la investigación cualitativa. Madrid. 


\section{CIENCIAMATRIA \\ Revista Interdisciplinaria de Humanidades, Educación, Ciencia y Tecnología \\ Año V. Vol. V. N8. Enero - Junio 2019 \\ Hecho el depósito de ley: pp201602FA4721 \\ ISSN-L: 2542-3029; ISSN: 2610-802X \\ Universidad Nacional Experimental Francisco de Miranda (UNEFM). Santa Ana de Coro. Venezuela \\ José Manuel Gómez ; Celimar Rodríguez}

12. IANNI, O. (1996) Teorías de la Globalización. Siglo XXI, Méjico.

13. MARTíNEZ, S. (1991). Investigación-acción y competencia docente. Revista Interuniversitaria de Formación de Profesorado.

14. PADRON, J. (1992) "PARADIGMAS" de investigación en ciencias sociales un enfoque curricular

15. PEÑA, K; PÉREZ, M Y RONDÓN, E (2010). Redes sociales en Internet: reflexiones sobre sus posibilidades... Revista de Teoría y Didáctica de las Ciencias Sociales. Mérida-Venezuela. ISSN 1316-9505. Enero-Diciembre. № 16 (2010): 173-205.

16. Panditt, N. (1995). La creación de teoría. Una aplicación reciente del método de teoría fundamentada. (A. R. Rojas P, Trad.). Informe Qualitativo, 2 (4).

17. PROYECTO NACIONAL SIMÓN BOLÍVAR (2007). Primer Plan Socialista 20072013. Presidencia de la República. Caracas.

18. SALINAS, J. (2004) Innovación docente y uso de las TIC en la enseñanza universitaria. Revista Universalidad y sociedad del conocimiento.

19. STRAUSS, A. (1970) Descubriendo nuevas teorías de teorías previas, Nueva Jersey: Prentice-Hall.

20.STRAUSS, A., y CORBIN, J. (2002). Bases de la investigación cualitativa. Técnicas y procedimientos para desarrollar la teoría fundamentada. Colombia: Editorial Universidad de Antioquia.

21. Rubio, J. (2005). El aporte de la tecnología informática al aprendizaje basado en problemas usando modelos de trabajo interactivos. Tesis doctoral de la Universidad de Sevilla.

22. SANTAMARÍA, F. (2005): "Herramientas colaborativas para la enseñanza usando tecnologías web: weblogs, wikis, redes sociales y web 2.0"

23. UNESCO (2009) La nueva dinámica de la educación superior y la investigación para el cambio social y el desarrollo. Conferencia Mundial sobre la Educación Superior- 2009, París.

24. LEY ORGÁNICA DE EDUCACIÓN (2009). Ediciones de la Presidencia de la República. 
25. OPSU (2009) PROYECTO NACIONAL DE EDUCACIÓN SUPERIOR A DISTANCIA Consejo Nacional de Universidades, Caracas.

26. VERGARA, V. (2009) Redes sociales y efecto de los pares como predictores del rendimiento escolar en alumnos de cuarto año básico de la comuna de concepción. Revista de Estudios y Experiencias en Educación, vol. 8, núm. 16, 2009, pp. 39-50 Universidad Católica de la Santísima Concepción Concepción, Chile

27. REQUENA, S. (1989) "El concepto de red social" en la Revista española deinvestigaciones sociológicas (REIS).

28. WEGNER, E. 2005. "Communities of practice, learning, meaning and identity". Cambriedge University Press.

C2019 por los autores. Este artículo es de acceso abierto y distribuido según los términos y condiciones de la licencia Creative Commons Atribución-NoComercial-Compartirlgual 4.0 Internacional (CC BY-NC-SA 4.0) (https://creativecommons.org/licenses/by-nc-sa/4.0/). 